\title{
Plasma code for astrophysical charge exchange emission at X-ray wavelengths
}

\author{
Liyi $\mathrm{Gu}^{1}$, Jelle Kaastra ${ }^{1,2}$, and A. J. J. Raassen ${ }^{1,3}$ \\ ${ }^{1}$ SRON Netherlands Institute for Space Research, Sorbonnelaan 2, 3584 CA Utrecht, The Netherlands \\ e-mail: L.Gu@sron.nl \\ 2 Leiden Observatory, Leiden University, PO Box 9513, 2300 RA Leiden, The Netherlands \\ 3 Astronomical Institute "Anton Pannekoek", University of Amsterdam, Science Park 904, 1098 XH Amsterdam, The Netherlands
}

Received 22 October 2015 / Accepted 22 January 2016

\begin{abstract}
Charge exchange X-ray emission provides unique insight into the interactions between cold and hot astrophysical plasmas. Besides its own profound science, this emission is also technically crucial to all observations in the X-ray band, since charge exchange with the solar wind often contributes a significant foreground component that contaminates the signal of interest. By approximating the cross sections resolved to $n$ and $l$ atomic subshells and carrying out complete radiative cascade calculation, we have created a new spectral code to evaluate the charge exchange emission in the X-ray band. Compared to collisional thermal emission, charge exchange radiation exhibits enhanced lines from large- $n$ shells to the ground, as well as large forbidden-to-resonance ratios of triplet transitions. Our new model successfully reproduces an observed high-quality spectrum of comet C/2000 WM1 (LINEAR), which emits purely by charge exchange between solar wind ions and cometary neutrals. It demonstrates that a proper charge exchange model will allow us to probe the ion properties remotely, including charge state, dynamics, and composition, at the interface between the cold and hot plasmas.
\end{abstract}

Key words. atomic data - atomic processes - comets: individual: C/2000 WM1 (LINEAR)

\section{Introduction}

Charge exchange (CX hereafter) occurs when an atom collides with a multicharged ion. It produces X-ray line emission if the ion charge is reasonably strong. The first discovery of CX X-ray emission in astronomical objects was made by observing the comet C/Hyakutake 1996 B2 with the ROSAT telescope (Lisse et al. 1996; Cravens 1997). This opened up a new research field to X-ray astronomers, and the related study soon expanded to various types of celestial objects. The CX process can partly explain the soft X-ray $(<2 \mathrm{keV})$ spectrum of the polar component in Jupiter and other planets in the solar system (e.g., Bhardwaj 2006; Branduardi-Raymont et al. 2007). As suggested in many papers, such as Cox (1998), Cravens (2000), Snowden et al. (2004), and Fujimoto et al. (2007), the geocoronal and heliospheric area is also glowing in soft X-rays by the CX between solar wind ions and interstellar neutral atoms. CX is also a potential mechanism for X-ray emission from the North Polar Spur region (Lallement 2009), stellar winds of supergiants (Pollock 2007), shock rims of supernova remnants (Katsuda et al. 2011; Cumbee et al. 2014), starburst galaxies (Tsuru et al. 2007; Liu et al. 2011), and even clusters of galaxies (Walker et al. 2015).

The astronomical discoveries of CX emission have also stimulated theoretical and laboratory studies of this process. Recent atomic calculations based on the quantum mechanical close coupling method (e.g., Wu et al. 2011; Nolte et al. 2012) have so far provided the most reliable estimate of the cross section for single electron capture in the low-velocity regime. Laboratory investigations broadened the species of neutral reactants from the hydrogen atom to various molecules (e.g., $\mathrm{CO}, \mathrm{H}_{2} \mathrm{O}$ ), to match the real conditions better, especially for comets (Bodewits et al. 2006; Wargelin et al. 2008).

To link the atomic and astrophysical work on the CX process, we present here a new spectral model that features the most recent atomic data and a complete radiative transition calculation. Another model for CX has been introduced by Smith et al. (2014), which is more focused on the transition calculation than on the actual reaction cross section. In Sect. 2, we describe the physical assumption of our model. In Sect. 3, we show in detail how the CX line emission is calculated from basic atomic data. In Sect. 4, we demonstrate our model by applying it to real data.

\section{Assumptions}

Our CX emission model is calculated based on three key assumptions. First, the charge transfer rates are obtained based on single electron capture in ion-neutral collision. Although multielectron neutral targets might be important for some environments (e.g., comets) where the channels of multi-electron capture do exist (e.g., Bodewits et al. 2006), the data available on such reactions are much less complete than those for single electron capture. As shown in the experimental results of, for example, Greenwood et al. (2001), single capture can provide a first-order approximation to multi-electron reactions. Secondly, as a related issue, we assume that the $\mathrm{CX}$ with atomic hydrogen is a reasonable representative of the real case, in terms of the cross section dependences on ion velocity and captured electron state (see details in Sect. 3). To correct for the helium atom, we 
Table 1. Collected charge exchange data.

\begin{tabular}{|c|c|c|c|c|c|c|c|}
\hline Ion & Method $^{a}$ & Type $^{b}$ & Reference & Ion & Method $^{a}$ & Type $^{b}$ & Reference \\
\hline $\mathrm{Be}^{4+}$ & UDWA & $n l$ & Ryufuku (1982) & $\mathrm{N}^{7+}$ & MOCC & $n l$ & Harel et al. (1998) \\
\hline $\mathrm{B}^{5+}$ & UDWA & $n l$ & Ryufuku (1982) & $\mathrm{O}^{8+}$ & MOCC & $n l$ & Harel et al. (1998) \\
\hline $\mathrm{C}^{6+}$ & UDWA & $n l$ & Ryufuku (1982) & $\operatorname{Ar}^{q^{+}}(15 \leq q \leq 18)$ & CTMC & $n l$ & Whyte et al. (1998) \\
\hline $\mathrm{O}^{8+}$ & UDWA & $n l$ & Ryufuku (1982) & $\mathrm{Ne}^{10+}$ & CTMC & $n$ & Perez et al. (2001) \\
\hline $\mathrm{O}^{8+}$ & MOCC & $n l$ & Shipsey et al. (1983) & $\mathrm{Ar}^{18+}$ & CTMC & $n$ & Perez et al. (2001) \\
\hline $\mathrm{Be}^{4+}$ & AOCC & $n l$ & Fritsch \& Lin (1984) & $\mathrm{Fe}^{18+}$ & СТMC & $n$ & Perez et al. (2001) \\
\hline $\mathrm{B}^{5+}$ & $\mathrm{AOCC}$ & $n l$ & Fritsch \& Lin (1984) & $\mathrm{O}^{\mathrm{q}^{+}}(5 \leq q \leq 8)$ & comp. & $n l$ & Raković et al. (2001) \\
\hline $\mathrm{C}^{4+}$ & AOCC & $n l$ & Fritsch \& Lin (1984) & $\mathrm{Li}^{3+}$ & CTMC & $n l$ & Errea et al. (2004) \\
\hline $\mathrm{C}^{6+}$ & $\mathrm{AOCC}$ & $n l$ & Fritsch \& Lin (1984) & $\mathrm{Ne}^{10+}$ & CTMC & $n l$ & Errea et al. (2004) \\
\hline $\mathrm{O}^{8+}$ & $\mathrm{AOCC}$ & $n l$ & Fritsch \& Lin (1984) & $\operatorname{Ar}^{q^{+}}(15 \leq q \leq 18)$ & CTMC & $n l$ & Schultz et al. (2010) \\
\hline $\mathrm{Fe}^{\mathrm{q}+}(5 \leq q \leq 26)$ & comp. & total & Phaneuf et al. (1987) & $\mathrm{N}^{6+}$ & QMOCC & $n l S$ & Wu et al. (2011) \\
\hline $\mathrm{C}^{\mathrm{q}+}(1 \leq q \leq 6)$ & comp. & total & Janev et al. (1988) & $\mathrm{O}^{6+}$ & QMOCC & $n l S$ & Wu et al. (2012) \\
\hline $\mathrm{O}^{\mathrm{q}+}(1 \leq q \leq 8)$ & comp. & total & Janev et al. (1988) & $\mathrm{C}^{5+}$ & QMOCC & $n l S$ & Nolte et al. (2012) \\
\hline $\mathrm{C}^{6+}$ & comp. & $n l$ & Janev et al. (1993) & $\mathrm{Fe}^{\mathrm{q}+}(25 \leq q \leq 26)$ & MCLZ & $n$ & Mullen et al. (2015) \\
\hline $\mathrm{O}^{8+}$ & comp. & $n l$ & Janev et al. (1993) & $\mathrm{He}^{2+}$ & EXP & total & Fite et al. (1962) \\
\hline $\mathrm{C}^{6+}$ & СТMC & $n l$ & Olson \& Schultz (1989) & $\mathrm{He}^{\mathrm{q}+}(1 \leq q \leq 2)$ & EXP & total & Olson et al. (1977) \\
\hline $\mathrm{O}^{8+}$ & CTMC & $n l$ & Olson \& Schultz (1989) & $\mathrm{O}^{\mathrm{q}+}(3 \leq q \leq 8)$ & EXP & total & Meyer et al. (1979) \\
\hline $\mathrm{Li}^{3+}$ & CDWA & $n$ & Belkić (1991) & $\mathrm{Si}^{9+}$ & EXP & total & Meyer et al. (1979) \\
\hline $\mathrm{Be}^{4+}$ & CDWA & $n$ & Belkić (1991) & $\mathrm{Fe}^{15+}$ & EXP & total & Meyer et al. (1979) \\
\hline $\mathrm{B}^{5+}$ & CDWA & $n$ & Belkić (1991) & $\mathrm{C}^{6+}$ & EXP & total & Meyer et al. (1985) \\
\hline $\mathrm{C}^{6+}$ & CDWA & $n l$ & Belkić (1991) & $\mathrm{N}^{\mathrm{q}^{+}}(6 \leq q \leq 7)$ & EXP & total & Meyer et al. (1985) \\
\hline $\mathrm{N}^{7+}$ & CDWA & $n$ & Belkić (1991) & $\mathrm{O}^{\mathrm{q}+}(7 \leq q \leq 8)$ & EXP & total & Meyer et al. (1985) \\
\hline $\mathrm{O}^{8+}$ & CDWA & $n l$ & Belkić (1991) & $\mathrm{F}^{\mathrm{q}+}(8 \leq q \leq 9)$ & EXP & total & Meyer et al. (1985) \\
\hline $\mathrm{He}^{2+}$ & MOCC & $n l$ & Harel et al. (1998) & $\mathrm{Ne}^{\mathrm{q}^{+}}(9 \leq q \leq 10)$ & EXP & total & Meyer et al. (1985) \\
\hline $\mathrm{Li}^{3+}$ & MOCC & $n l$ & Harel et al. (1998) & $\mathrm{B}^{\mathrm{q}+}(3 \leq q \leq 5)$ & EXP & total & Crandall et al. (1979) \\
\hline $\mathrm{Be}^{4+}$ & MOCC & $n l$ & Harel et al. (1998) & $\mathrm{C}^{\mathrm{q}+}(3 \leq q \leq 4)$ & EXP & total & Crandall et al. (1979) \\
\hline $\mathrm{B}^{5+}$ & MOCC & $n l$ & Harel et al. (1998) & $\mathrm{N}^{\mathrm{q}^{+}}(3 \leq q \leq 4)$ & EXP & total & Crandall et al. (1979) \\
\hline$C^{6+}$ & MOCC & $n l$ & Harel et al. (1998) & $\mathrm{O}^{\mathrm{q}+}(5 \leq q \leq 6)$ & EXP & total & Crandall et al. (1979) \\
\hline
\end{tabular}

Notes. ${ }^{(a)}$ Methods include: UDWA (unitarized distorted-wave approximation), MOCC (molecular-orbital close-coupling), AOCC (atomic-orbital close-coupling), comp. (data compilation), CTMC (classical trajectory Monte Carlo), CDWA (continuum distorted-wave approximation), QMOCC (quantum molecular-orbital close-coupling), MCLZ (multichannel Landau-Zener), and EXP (experiment); ${ }^{(b)}$ Data types are: total (total cross section only), $n$ (principle quantum number $n$-resolved cross section), $n l$ ( $n l$-resolved), and $n l S$ ( $n l S$-resolved).

approximate the helium cross section using the scaling rule of Janev \& Gallagher (1984),

$\frac{\sigma_{\mathrm{He}}}{\sigma_{\mathrm{H}}}=\frac{N_{\mathrm{He}}}{N_{\mathrm{H}}}\left(\frac{I_{\mathrm{H}}}{I_{\mathrm{He}}}\right)^{2}$,

where $N_{\mathrm{He}}$ and $N_{\mathrm{H}}$ are the numbers of valence electrons, and $I_{\mathrm{He}}$ and $I_{\mathrm{H}}$ are the ionization potentials. For plasmas with cosmological abundances (10\% and $90 \%$ in number for helium and hydrogen, respectively), the combined CX cross section can be derived from the pure hydrogen value by $\sigma=0.96 \sigma_{\mathrm{H}}$.

As the third assumption, the radiative processes related to free electrons, e.g., collisional excitation and radiative/dielectron recombination, are ignored in the modeling. It will prevent the CX-induced transitions, which usually involves large- $n$ shells (Sect. 3.2), from being overpowered in emission by the collisional excitation dominating the small- $n$ shells. This assumption can be validated because the ionic $\mathrm{CX}$ has much larger cross section than the electronic processes at X-ray energies.

\section{Method}

To calculate CX line emission, we first determine the ion state population after electron capture, and then solve the possible radiative cascading pathways to the ground state. The first step can be further divided into three components, i.e., the total capture cross sections, and the cross sections into each $n$ - and $l$-resolved level.
The main challenge is that the current atomic data for $n$ and $l$-resolved cross sections are far from complete. It is therefore necessary to investigate the available data for intrinsic scaling relations among $n$ - and $l$-resolved parameters, as described in Sects. 3.2 and 3.3, respectively. In practice, we collected all available cross sections from the literature and applied the derived scaling relations when the actual data are absent.

\subsection{Total cross sections}

It is commonly believed that CX has a very large cross section compared to electronic processes, typically of about $10^{-15}-10^{-14} \mathrm{~cm}^{-2}$. To obtain the cross section as a function of collision velocity $v$, we compiled the results reported in previous theoretical calculations (Ryufuku 1982; Shipsey et al. 1983; Fritsch \& Lin et al. 1984; Phaneuf et al. 1987; Janev et al. 1988, 1993; Olson \& Schultz 1989; Belkić 1991; Harel et al. 1998; Whyte et al. 1998; Perez et al. 2001; Errea et al. 2004; Schultz et al. 2010; Wu et al. 2011; Nolte et al. 2012) and experimental measurements (Fite et al. 1962; Olson et al. 1977; Meyer et al. 1979, 1985; Crandall et al. 1979). A complete list of the data sources is shown in Table 1. For the theoretical calculations, we employed a practical criterion reported in Janev et al. (1993), which depends on the calculation method, to assess the energy range of validity.

As shown in Wargelin et al. (2008), for example, the average cross sections usually exhibit a linear dependence on ion charge $q$. Such a feature is also seen in Fig. 1. In addition, due 


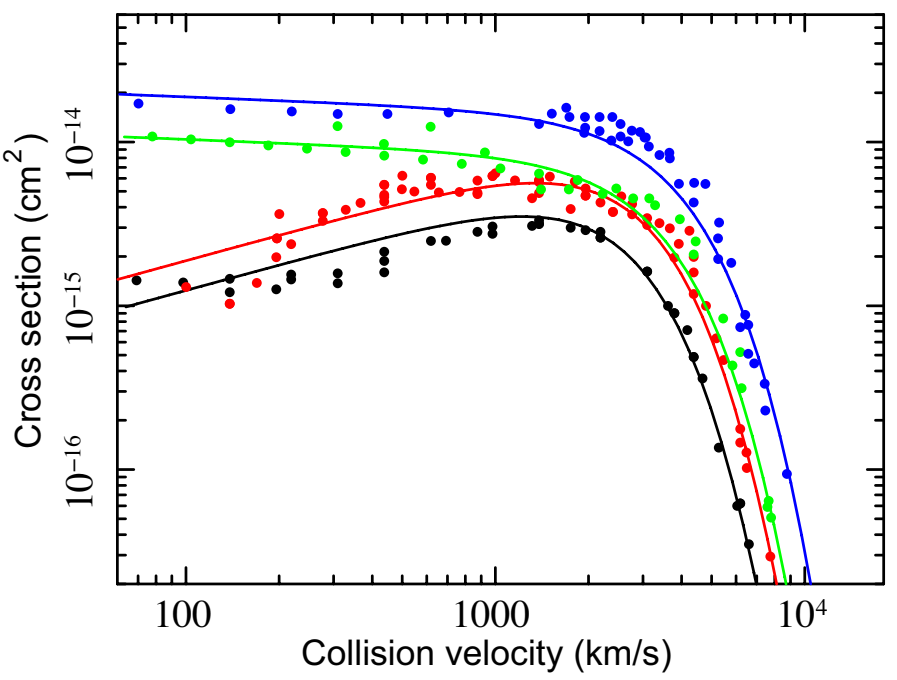

Fig. 1. Total cross sections, as functions of collision velocity, for $\mathrm{B}^{5+}$ (black), $\mathrm{O}^{8+}$ (red), $\mathrm{Ne}^{10+}$ (green), and $\mathrm{Ar}^{18+}$ (blue) interacting with a hydrogen atom. The data points are actual values from previous calculations, and solid lines are the scaling law described in Sect. 3.1.

to the non-resonant effect (e.g., Janev \& Winter 1985), the cross sections for small- $q$ reactions, e.g., $\mathrm{B}^{5+}$ with the $\mathrm{H}$ atom, exhibit a maximum at certain $v \leq v_{0}$ (where $v_{0}$ is the orbital velocity of bound electrons), and exponential decrease toward low energy. For large- $q$ ions, such as $\mathrm{Ne}^{10+}$ and $\mathrm{Ar}^{18+}$, this effect diminishes as the number of channels for resonant reactions becomes large. In the high energy regime $\left(v>v_{0}\right)$, both small- $q$ and large- $q$ reactions fall off as charge transfer into discrete levels become strongly coupled with the continuum, and the ionization process starts to dominate. To combine the $q$ - and $v$-dependence, we use a scaling law refined from Janev \& Smith (1993),

$\sigma=a_{1} q E_{\mathrm{s}}^{a_{2}} \ln \left(\frac{a_{3}}{E_{\mathrm{s}}}+a_{4}\right)\left(1+\frac{E_{\mathrm{s}}}{a_{5}}\right)^{a_{6}}$,

where $E_{\mathrm{s}}=E / q^{0.43}$ is a scaled version of the collision energy $E$ given in $\mathrm{eV} / \mathrm{amu}$, and $a_{1}$ to $a_{6}$ are $q$-dependent fitting parameters. The average best-fit values for $a_{1}$ to $a_{6}$ are $(4.6,1.0,0.2$, $1.0,83 .,-8.9)$ and $(0.3,0.1,1 ., 10 ., 158 .,-9.9)$, for $3<q<10$ and $q \geq 10$, respectively. As shown in Fig. 1, the scaling relation in general reproduces the cross sections of all ions well. Some residuals are seen at $v \leq 500 \mathrm{~km} \mathrm{~s}^{-1}$, where the data oscillate around the fitting curve for small- $q$ species. This is probably caused by the discrete nature of product ion energy levels (e.g., Ryufuku 1980). In practice, since the actual data for such small$q$ species are usually available in the literature, the bias on the scaling relation can barely affect our model. Equation (2) is then used to calculate cross sections for ions that are still missing in previous publications.

\section{2. $n$-shell populations}

The CX probability reaches its maximum when the two energy states, before and after the transition, are closest to each other. For low-speed collisions $\left(v \ll v_{0}\right)$, the potential energy dominates the interaction, and the principle quantum number $n_{\mathrm{p}}$ of the most populated energy level can be written by

$n_{\mathrm{p}}=\sqrt{\frac{I_{\mathrm{H}}}{2 I_{\mathrm{t}}}} q\left(1+\frac{q-1}{\sqrt{2 q}}\right)^{-0.5}$
(Janev \& Winter 1985), where $I_{\mathrm{H}}$ and $I_{\mathrm{t}}$ are the ionization potentials of $\mathrm{H}$ and the target atom, respectively. This means that the peak level is determined by the combined potential of the projectile ion and target atom. For most ions, $n_{\mathrm{p}}$ is much larger than unity. In the high-speed regime $\left(v \sim v_{0}\right)$, the collision dynamics become more important for the capture process, and the peak population level is gradually smeared out among several adjacent shells.

We compiled the velocity-dependent, $n$-resolved cross sections for reactions involving $\mathrm{Be}, \mathrm{B}, \mathrm{C}, \mathrm{N}, \mathrm{O}, \mathrm{Ne}$, and Fe ions from theoretical calculations (Ryufuku 1982; Shipsey et al. 1983; Fritsch \& Lin et al. 1984; Belkić et al. 1992; Janev et al. 1993; Toshima \& Tawara 1995; Harel et al. 1998; Raković et al. 2001; Errea et al. 2004; Nolte et al. 2012; Wu et al. 2011, 2012; Mullen et al. 2015). As shown in Fig. 2, the capture cross sections are normalized to the total value of each ion and plotted against $\left(n-n_{\mathrm{p}}\right) / n_{\mathrm{p}}$. This puts them on a roughly similar $n$-distribution function, which reconfirms the result in Janev \& Winter (1985). The velocity dependence of the $n$-populations also agrees with the consensus described above. As seen in Fig. 2, for each velocity, we fit the $n$-distribution by a phenomenological third-degree polynomial curve, which reproduces the data. Some minor deviations, typically a few percent of the total cross section, are seen for low-capture shells. The same fitting was done for ten other velocity points to fully cover the range of $50 \leq v \leq 5000 \mathrm{~km} \mathrm{~s}^{-1}$. Details of the fitting procedure can be found in Appendix A. As a first-order approximation, we assumed that all the CX processes with hydrogen-atom targets follow the same profile in the $\left(n-n_{\mathrm{p}}\right) / n_{\mathrm{p}}$ versus $\sigma$ space and have a similar velocity dependence. In this way, the $n$-resolved populations were calculated for all the rest ions. When applying the $n$-distribution to astrophysical plasmas contaminated by helium, the actual peak $n$ would be slightly lower than the calculated value, since the helium target has a larger ionization potential than atomic hydrogen. Assuming the cosmic abundances, this would lead to an uncertainty of $\leq 10 \%$ for the obtained $n$-resolved cross sections.

\subsection{I-subshell populations}

Besides the $n$-dependent capture, it is known that the CX process also exhibits strong selective properties with respect to the final electron orbit angular momentum $l$. As discussed in Janev $\&$ Winter (1985) and Suraud et al. (1991), for example, the $l$-selectivity is very sensitive to the collision velocity and is often governed by high-order processes in the transition (e.g., rotational mixing). Typically, the $l$ distribution is approximated as a function of $n, l$, and $q$ in at least five forms shown as follows:

$$
\begin{aligned}
& W_{n}^{11}(l)=(2 l+1) \frac{[(n-1) !]^{2}}{(n+l) !(n-l-1) !} \quad(\text { low energy I), } \quad(4) \\
& \left.W_{n}^{12}(l)=l(l+1)(2 l+1) \frac{(n-1) !(n-2) !}{(n+l) !(n-l-1) !} \quad \text { (low energy II }\right), \quad(5)
\end{aligned}
$$

$W_{n}^{\mathrm{se}}(l)=\left(\frac{2 l+1}{q}\right) \exp \left[-\frac{l(l+1)}{q}\right]$

(separable),

$W_{n}^{\mathrm{ev}}(l)=1 / n$

(even),

$W_{n}^{\mathrm{st}}(l)=(2 l+1) / n^{2}$

(statistical). 


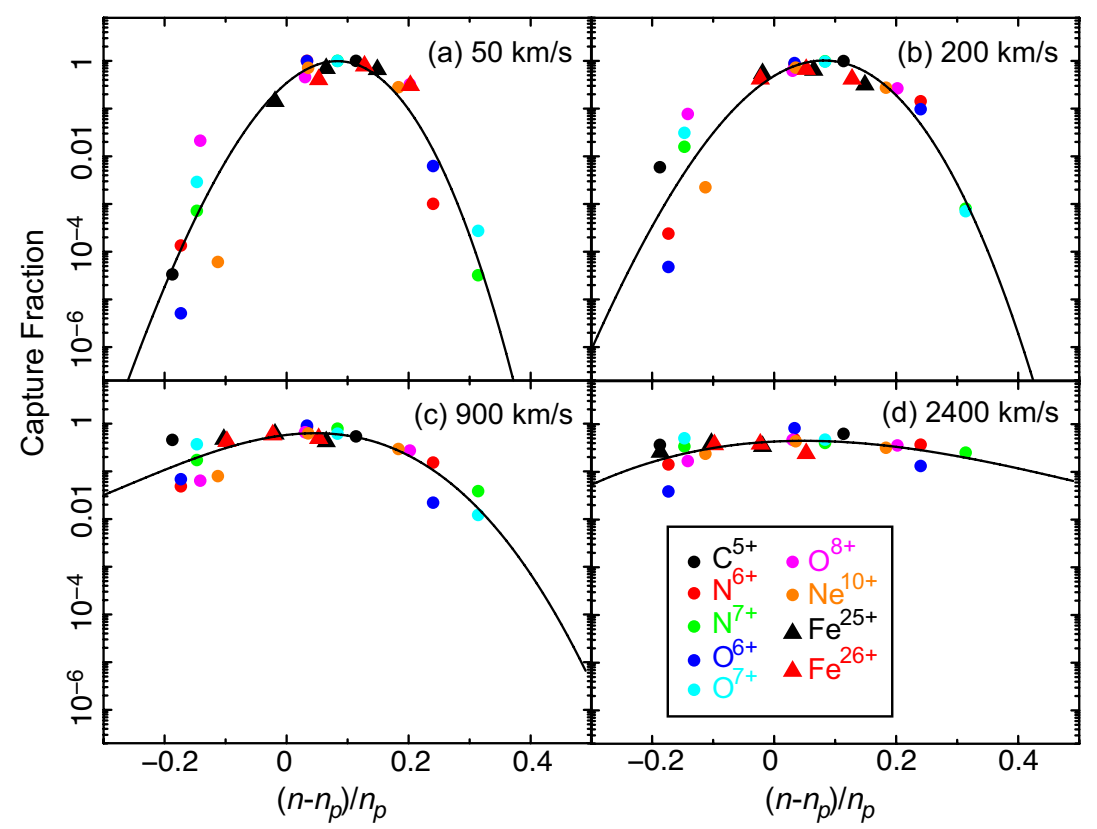

$200 \mathrm{~km} / \mathrm{s}$

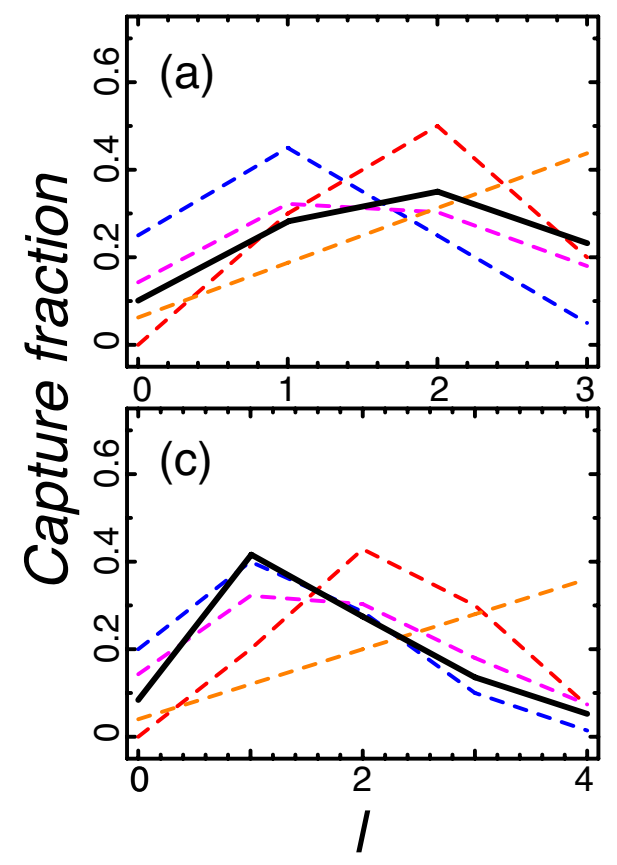

$2000 \mathrm{~km} / \mathrm{s}$

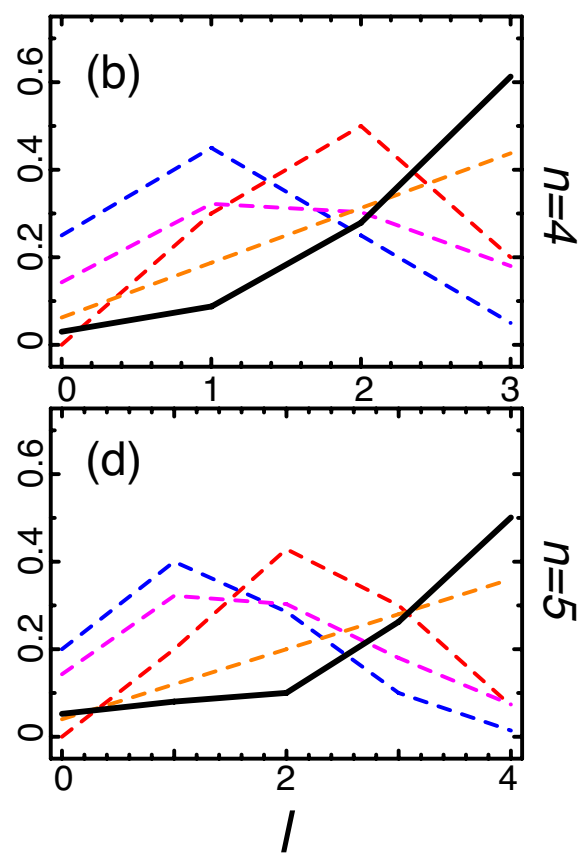

Fig. 2. Fractions of single electron capture into $n$ shell, at $v=50 \mathrm{~km} \mathrm{~s}^{-1}$ a); $200 \mathrm{~km} \mathrm{~s}^{-1} \mathbf{b}$ ); $900 \mathrm{~km} \mathrm{~s}^{-1} \mathbf{c}$ ); and $2400 \mathrm{~km} \mathrm{~s}^{-1} \mathbf{d}$ ); as a function of $\left(n-n_{\mathrm{p}}\right) / n_{\mathrm{p}}$. Different sets of data points represent different projectile ions, as indicated in d); and the solid lines are the polynomial fitting to the data.

Fig. 3. Averaged fractions of $l$-dependent capture for $\mathrm{N}^{7+}$ and $\mathrm{O}^{7+}$, plotted as a function of $l$. Black solid lines are the data from theoretical calculations, and the red, blue, magenta, and orange dashed lines are empirical functions shown in Eqs. (4)-(6), (8), respectively. Panels a) and b) are the capture for $n=4$ at $v=200 \mathrm{~km} \mathrm{~s}^{-1}$ and $2000 \mathrm{~km} \mathrm{~s}^{-1}$, respectively, and c) and d) are those for $n=5$.

In the low velocity regime $\left(v \ll v_{0}\right)$, generally the transferred electron does not carry sufficient angular momentum to populate large- $l$ subshells. These electrons form a peak at $l=1$ or 2 (e.g., Abramov et al. 1978), which can be roughly described by functions $W^{11}, W^{12}$, and/or $W^{\text {se }}$. As the collision velocity increases, the $l$ peak is gradually smeared out by rotational mixing of the coupling Stark states, and the subshells are populated according to the statistical weight $W^{\text {st }}$.

It is clear that none of the five functions alone can describe the $l$-populations for all velocities. Previous work further suggested that the choice of weighting function also depends on the principle quantum number $n$; the $l$-distributions of $n \leq n_{\mathrm{p}}$ subshells are often found to be different from those with $n>n_{\mathrm{p}}$ (e.g., Janev \& Winter 1985). To elucidate the $v$ - and $n$-dependence, we plot in Figs. $3 \mathrm{a}$ and $\mathrm{c}$ the averaged l-distributions at $v=200 \mathrm{~km} \mathrm{~s}^{-1}$ for $\mathrm{N}^{7+}$ and $\mathrm{O}^{7+}$ ions, based on data from theoretical calculations (Belkić et al. 1992; Toshima \& Tawara 1995; Raković et al. 2001). The two dominant shells with $n=4$ and 5 are used to represent the $n \leq n_{\mathrm{p}}$ and $n>n_{\mathrm{p}}$ groups, respectively. This is valid since the rest shells contribute less than $0.1 \%$ of the total rate at $v=200 \mathrm{~km} \mathrm{~s}^{-1}$. The $\mathrm{N}^{7+}$ and $\mathrm{O}^{7+}$ ions exhibit a large capture fraction into $l=1$ for $n=5$, while for $n=4$, subshells with $l=2$ and 3 are equally or even more populated than $l=1$. By comparing data with the five weighting functions, we found that the $l$-populations of the two ions are best approximated by the $W^{12}$ function at $n=5$, while the $n=4$ shells resemble the $W^{\text {se }}$ function more. As shown in Figs. $3 b$ and d, the same calculation was done at $v=2000 \mathrm{~km} \mathrm{~s}^{-1}$, where the two distributions become roughly consistent and match the statistical weight $W^{\text {st }}$ best. By incorporating the data of other available 
ions, we determined the preferred weighting function, dependent on $v$ and $n$, and applied it to the rest ions (see Appendix B for details).

\subsection{Spectral model}

The CX emission line is produced when the captured electron relaxes to the valence shell of the ground state of the product ion. To perform the complete cascade calculation, we obtained the energies and transition probabilities for all the atomic levels up to $n=16$, which exceeds the maximum capture state for all ions used in the code (e.g., maximum capture $n=12$ and 13 for $\mathrm{Fe}^{25+}+\mathrm{H}$ collision). The FAC code for theoretical atomic calculations ( $\mathrm{Gu} 2008$ ) was used as a baseline tool, and the energies were calibrated to those derived with another atomic structure code (Cowan 1981), as well as the experimental measurements at the National Institute of Standards and Technology ${ }^{1}$. The line spectrum was then calculated from the cascade network given the source term of the CX rates. More features of the new CX model are described in Appendix C.

As described in Sect. 3.2, the CX process can populate the large- $n$ shells, so line emission from such shells is strongly enhanced compared to collisional thermal radiations. For instance, the $\mathrm{O}$ VIII Ly $\delta$ line at $14.8 \AA$ is stronger than the Ly $\gamma$ line at $15.2 \AA$. Similar conditions can be found in many other transitions: e.g., the O VII He $\delta$ line at $17.4 \AA$, the N VII Ly $\delta$ line at $19.4 \AA$, and C VI Ly $\delta$ line at $26.4 \AA$. The derived CX spectrum also features a large $\mathrm{G}$ ratio, the forbidden plus intercombination lines to resonance line ratio, since the collisional excitation process becomes negligible in CX plasmas (Sect. 2).

\subsection{Bias from systematic weight}

As reported recently by Nolte et al. (2012), the singlet-to-triplet ratio of the $\mathrm{C}^{4+}$ ion, produced from a low-velocity $\mathrm{C}^{5+}+\mathrm{H}$ reaction, covers a broad range of 0.01-100 for different $n, l$, and $v$. The apparent bias from the commonly adopted statistical value 3 is probably caused by electron-electron interaction during the capture. A similar effect was also reported in other papers (e.g., Wu et al. 2011 for $\mathrm{N}^{6+}+\mathrm{H}$ reaction), indicating that it could be a common property. Although the current data are still too sparse to fully implement the $S$-dependence, it is vital to estimate the induced biases on emission line ratios. As shown in Fig. 4, we compare the $\mathrm{G}$ ratio calculated based on the data from Nolte et al. (2012) with that assuming statistical weight for the $\mathrm{C}^{5+}+\mathrm{H}$ reaction. The largest bias is seen at $v \sim 500 \mathrm{~km} \mathrm{~s}^{-1}$, where the statistical weight is underestimated by about $30 \%$. The two ratios become roughly consistent at low and high velocity ends. The figure suggests that the current code can cover the actual $G$ ratio range, although the derived velocity would have a systematic error up to $200-300 \mathrm{~km} \mathrm{~s}^{-1}$.

\section{Real data fitting}

To verify the CX model, we fit it to the real data of comet $\mathrm{C} / 2000$ WM1 (LINEAR) observed by the XMM-Newton Reflection Grating Spectrometer (RGS), which has a high spectral resolution $\sim 0.07 \AA$ in soft X-rays (5-38 $\AA$ ). Comets are the favorite target for our purpose, since they emit bright X-rays that are produced exclusively by the CX between the highly charged heavy ions in the solar wind and cometary atmosphere (Dennerl 2010).

\footnotetext{
1 http://www.nist.gov/pml/data/asd.cfm
}

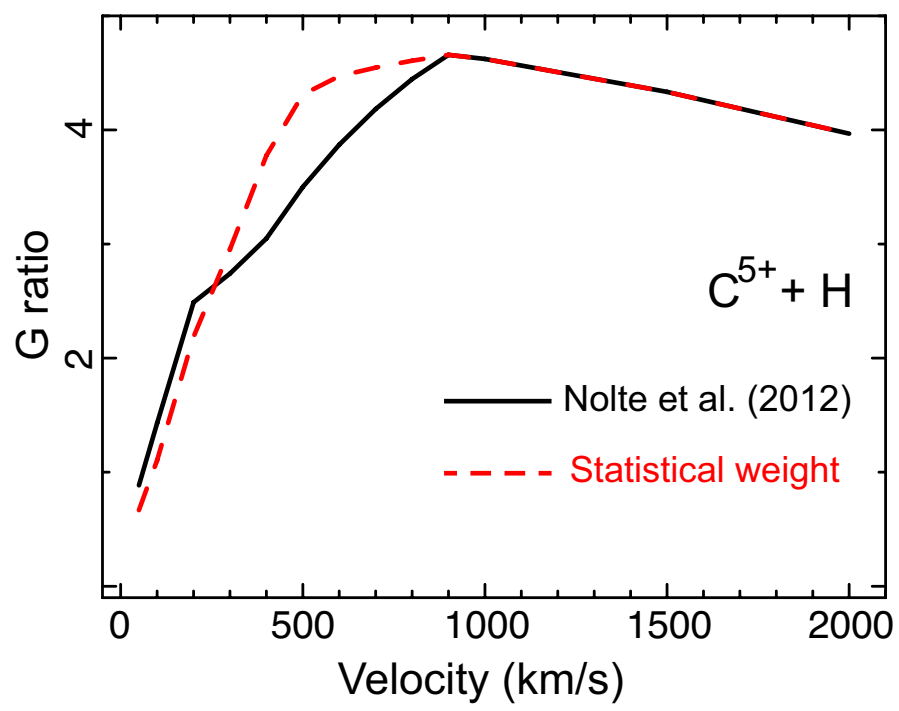

Fig. 4. G ratio of $\mathrm{CvHe} \alpha$ triplets, calculated based on the triplet-tosinglet ratios reported in Nolte et al. (2012, black) and the value given by statistical weight (red).

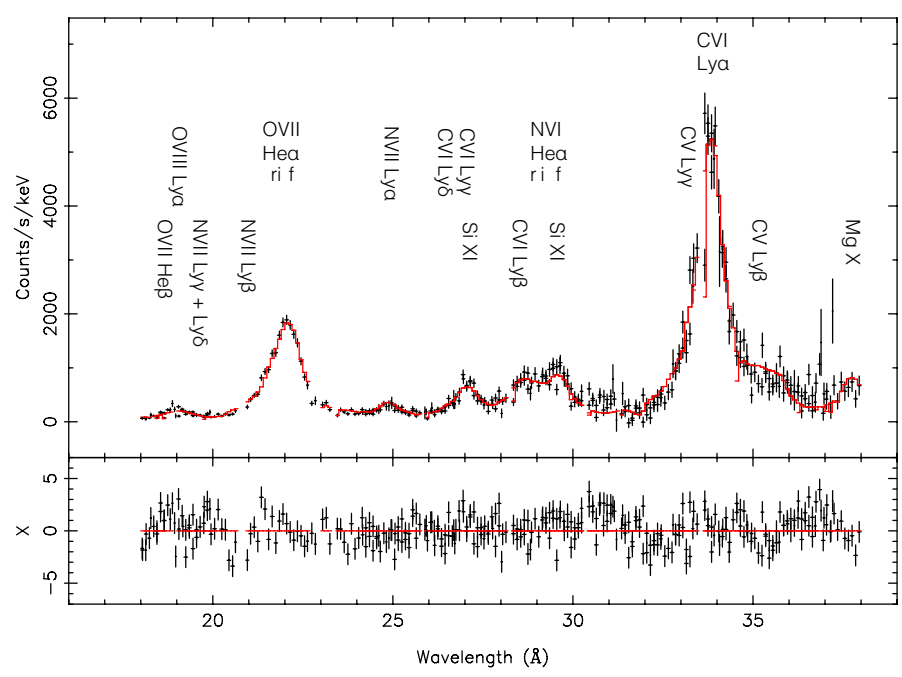

Fig. 5. CX fitting (red) to the RGS spectrum (black) of comet C/2000 WM1 (LINEAR).

As a caveat, the current model is based on an atomic hydrogen target, while the cometary neutrals are mainly moleculars, such as $\mathrm{H}_{2} \mathrm{O}$ and $\mathrm{CO}$. As reported in Bodewits et al. (2007) and Mullen et al. (2015), the cross sections of moleculars can be roughly approximated by that of $\mathrm{H}$ at intermediate and high velocities, while at low velocity $\left(v \lesssim 100 \mathrm{~km} \mathrm{~s}^{-1}\right)$, they become different by orders of magnitude. The cometary CX model with molecular targets will be reported in a subsequent paper (Mullen et al., in prep.).

\subsection{Observation}

Comet C/2000 WM1 was observed by XMM-Newton on December 2001 for a continuous exposure of about $62 \mathrm{ks}$. The RGS event files were created by using SAS 14.0 and the most recent calibration files. To remove events outside the field of view along the cross-dispersion direction $\left(5^{\prime}\right)$, we used only the $18 \mathrm{ks}$ exposure when the comet was close to the center point of the detector. The background component was approximated by the model background spectrum calculated by SAS tool rgsproc. 
To correct the spectral broadening due to spatial extent of the cometary X-ray halo, we used a 7-30 A image that was observed at the same period with the MOS1 detector of the European Photon Imaging Cameras (EPIC) onboard XMM-Newton. The final RGS data has more than 18000 counts recorded with high spectral resolution, making it the best X-ray data so far for the cometary CX study.

\subsection{Results}

Our study focuses on the $22-38 \AA(0.33-0.56 \mathrm{keV})$ band of the RGS spectrum. In the fits, we assume that all the solar wind ions have a same ionization temperature and collided with the comet atmosphere at a constant velocity during the exposure. The temperature and velocity are allowed to vary freely. We can also measure the $\mathrm{C}, \mathrm{N}, \mathrm{O}, \mathrm{Mg}$, and $\mathrm{Si}$ abundances of the wind, but other elements are not visible in the RGS energy band. Following the recipe of von Steiger et al. (2000), the solar abundances are set to the photospheric values from Grevesse \& Sauval (1998). The CX emissivity, which is determined by combining the ion and neutral densities, was also set free, although it would degenerate with the metal abundances to some extent. As shown in Fig. 5, the RGS spectrum can be fit reasonably well by one CX component, characterized by an ionization temperature of $0.14 \pm 0.01 \mathrm{keV}$ and a collision velocity of $200 \pm \pm_{45}^{85} \mathrm{~km} \mathrm{~s}^{-1}$. The fitting $\mathrm{C}$ statistic is 401 for a degree of freedom of 288 . The collision velocity is measured primarily by the strong $\mathrm{C}$ VI lines from different $n$ shells, including the Ly $\alpha$ line at $33.7 \AA$, the Ly $\beta$ line at $28.5 \AA$, the Ly $\gamma$ line at $27.0 \AA$, and the $\operatorname{Ly} \delta$ line at $26.4 \AA$. The $\mathrm{N}$ VII and $\mathrm{O}$ VII series also help in the velocity measurement. The smaller lower velocity error compared to the upper one is probably because the line ratios become more sensitive to the velocities at lower collision energies. The best-fit abundance ratios relative to $\mathrm{O}(\mathrm{C} / \mathrm{O}, \mathrm{N} / \mathrm{O}, \mathrm{Mg} / \mathrm{O}$, and $\mathrm{Si} / \mathrm{O})$ are measured to be $1.9 \pm 0.3,1.6 \pm 0.6,5 \pm 4$, and $3 \pm 2$, respectively. These values are roughly consistent with the average solar wind abundances reported in von Steiger et al. (2000).

The derived CX component appears to resemble the slowtype solar wind. It is well known that the slow wind is launched with a typical ionization state of $0.12-0.14 \mathrm{keV}$, which is quite different from that of a fast wind $(0.07 \mathrm{keV}$, Feldman et al. 2005). The ionization temperature remains nearly the same in the wind propagation, since the ionization/recombination timescales are often much longer than the travel time. On the other hand, according to the records of Advanced Composition Explorer (ACE), the solar wind ion speed at the Earth Lagrangian point $\mathrm{L} 1$ is in the range of $250-350 \mathrm{~km} \mathrm{~s}^{-1}$, in the period of the C/2000 WM1 observation by XMM-Newton. Since the heliocentric distance of the comet was nearly $1 \mathrm{AU}$, the ion speed at its environment should be close to the ACE record (Neugebauer et al. $2000)$. Consider that the comet had a velocity of $\sim 50 \mathrm{~km} \mathrm{~s}^{-1}$ relative to the Sun, the best-fit collision velocity measured with our CX model appears to be slightly lower than the wind speed in the comet restframe. This agrees with the picture presented in Bodewits et al. (2007), among others, in which the solar wind is somewhat decelerated in the comet bow shock region.

\section{Summary}

We developed a new plasma code to calculate charge exchange emission in X-ray band. To overcome the incompleteness in atomic data of cross sections, we derived scaling laws to approximate the $n$ - and $l$-distributions for various collision velocities in the range of $50-5000 \mathrm{~km} \mathrm{~s}^{-1}$. The radiative cascading calculation shows characteristic charge exchange emission features, including both high-shell transition lines and large $\mathrm{G}$ ratios of triplets. Our CX model successfully reproduces an observed high resolution X-ray spectrum from comet C/2000 WM1 with reasonable ionization temperature and collision velocity of the solar wind ions.

Acknowledgements. SRON is supported financially by NWO, the Netherlands Organization for Scientific Research.

\section{References}

Abramov, V. A., Baryshnikov, F. F., \& Lisitsa, V. S. 1978, Sov. J. Exp. Theor. Phys. Lett., 27, 464

Belkić, D. 1991, Phys. Scr, 43, 561

Belkić, D., Gayet, R., \& Salin, A. 1992, Atom. Data Nucl. Data Tables, 51, 59

Bhardwaj, A. 2006, Advances in Geosciences, Planetary Science (PS) (World Scientific Co, Pte. Ltd.), 215

Branduardi-Raymont, G., Bhardwaj, A., Elsner, R. F., et al. 2007, A\&A, 463, 761

Bodewits, D., Hoekstra, R., Seredyuk, B., et al. 2006, ApJ, 642, 593

Bodewits, D., Christian, D. J., Torney, M., et al. 2007, A\&A, 469, 1183

Cowan, R. D. 1981, Los Alamos Series in Basic and Applied Sciences (Berkeley: University of California Press)

Cox, D. P. 1998, Lect. Notes Phys., 506, 121

Crandall, D. H., Phaneuf, R. A., \& Meyer, F. W. 1979, Phys. Rev. A, 19, 504

Cravens, T. E. 1997, Geophys. Res. Lett., 24, 105

Cravens, T. E. 2000, ApJ, 532, L153

Cumbee, R. S., Henley, D. B., Stancil, P. C., et al. 2014, ApJ, 787, L31

Dennerl, K. 2010, Space Sci. Rev., 157, 57

Errea, L. F., Illescas, C., Méndez, L., et al. 2004, J. Phys. B At. Mol. Phys., 37, 4323

Feldman, U., Landi, E., \& Schwadron, N. A. 2005, J. Geophys. Res. (Space Phys.), 110, A07109

Fite, W. L., Smith, A. C. H., \& Stebbings, R. F. 1962, Roy. Soc. London Proc. Ser. A, 268, 527

Fritsch, W., \& Lin, C. D. 1984, Phys. Rev. A, 29, 3039

Fujimoto, R., Mitsuda, K., Mccammon, D., et al. 2007, PASJ, 59, 133

Greenwood, J. B., Williams, I. D., Smith, S. J., \& Chutjian, A. 2001, Phys. Rev. A, 63, 062707

Grevesse, N., \& Sauval, A. J. 1998, Space Sci. Rev., 85, 161

Gu M. F. 2008, Can. J. Phys., 86, 675

Harel, C., Jouin, H., \& Pons, B. 1998, Atom. Data Nucl. Data Tables, 68, 279

Janev, R. K., \& Gallagher, J. W. 1984, J. Phys. Chem. Ref. Data, 13, 1199

Janev, R. K., \& Smith, J. J. 1993, Atomic and Plasma-Material Interaction Data for Fusion (Supplement to the journal Nuclear Fusion), 4, 1

Janev, R. K., \& Winter, H. 1985, Phys. Rep., 117, 265

Janev, R. K., Phaneuf, R. A., \& Hunter, H. T. 1988, Atom. Data Nucl. Data Tables, 40, 249

Janev, R. K., Phaneuf, R. A., Tawara, H., \& Shirai, T. 1993, Atom. Data Nucl. Data Tables, 55, 201

Kaastra, J. S., Mewe, R., \& Nieuwenhuijzen, H. 1996, in 11th Colloq. on UV and X-ray Spectroscopy of Astrophysical and Laboratory Plasmas, 411

Katsuda S., Tsunemi, H., Mori, K., et al. 2011, ApJ, 730, 24

Lallement, R. 2009, Space Sci. Rev., 143, 427

Lisse, C. M., Dennerl, K., Englhauser, J., et al. 1996, Science, 274, 205

Liu, J., Mao, S., \& Wang, Q. D. 2011, MNRAS, 415, L64

Meyer, F. W., Phaneuf, R. A., Kim, H. J., Hvelplund, P., \& Stelson, P. H. 1979, Phys. Rev. A, 19, 515

Meyer, F. W., Howald, A. M., Havener, C. C., \& Phaneuf, R. A. 1985, Phys. Rev. A, 32, 3310

Mullen, P. D., Cumbee, R., Lyons, D., Stancil, P. C., \& B. J. Wargelin 2015, AAS Meet. Abst., 225, \#407.02

Neugebauer, M., Cravens, T. E., Lisse, C. M., et al. 2000, J. Geophys. Res., 105, 20949

Nolte, J. L., Stancil, P. C., Liebermann, H. P., et al. 2012, J. Phys. B At. Mol. Phys., 45, 245202

Olson, R. E., \& Schultz, D. R. 1989, Phys. Scr. T, 28, 71

Olson, R. E., Salop, A., Phaneuf, R. A., \& Meyer, F. W. 1977, Phys. Rev. A, 16, 1867

Perez, J. A., Olson, R. E., \& Beiersdorfer, P. 2001, J. Phys. B At. Mol. Phys., 34, 3063 
Phaneuf, R. A., Janev, R. K. \& Pindzola, M. S. 1987, Collisions of carbon and oxygen ions with electrons, H, H2 and He (Springfield, Va.: Controlled Fusion Atomic Data Center) Available from National Technical Information Service, Atomic data for fusion, 0888-8345; vol. 5. Controlled Fusion Atomic Data Center (Oak Ridge National Laboratory)

Pollock, A. M. T. 2007, A\&A, 463, 1111

Raković, M. J., Wang, J., Schultz, D. R., \& Stancil, P. C. 2001, AGU Spring Meeting Abstracts, 41

Ryufuku, H. 1982, Phys. Rev. A, 25, 720

Ryufuku, H., Sasaki, K., \& Watanabe, T. 1980, Phys. Rev. A, 21, 745

Schultz, D. R., Lee, T.-G., \& Loch, S. D. 2010, J. Phys. B At. Mol. Phys., 43, 144002

Shipsey, E. J., Green, T. A., \& Browne, J. C. 1983, Phys. Rev. A, 27, 821

Smith, R. K., Foster, A. R., Edgar, R. J., \& Brickhouse, N. S. 2014, ApJ, 787, 77

Snowden, S. L., Collier, M. R., \& Kuntz, K. D. 2004, ApJ, 610, 1182
Suraud, M. G., Hoekstra, R., de Heer, F. J., Bonnet, J. J., \& Morgenstern, R. 1991, J. Phys. B At. Mol. Phys., 24, 2543

Toshima, N., \& Tawara, H. 1995, NIFS-DATA-026, www.nifs.ac.jp/ report/nifs-data026.html

Tsuru, T. G., Ozawa, M., Hyodo, Y., et al. 2007, PASJ, 59, 269

von Steiger, R., Schwadron, N. A., Fisk, L. A., et al. 2000, J. Geophys. Res., 105, 27217

Walker, S. A., Kosec, P., Fabian, A. C., \& Sanders, J. S. 2015, MNRAS, 453, 2480

Wargelin, B. J., Beiersdorfer, P., \& Brown, G. V. 2008, Can. J. Phys., 86, 151

Whyte, D. G., Isler, R. C., Wade, M. R., et al. 1998, Phys. Plasmas, 5, 3694

Wu, Y., Stancil, P. C., Liebermann, H. P., et al. 2011, Phys. Rev. A, 84, 022711

Wu, Y., Stancil, P. C., Schultz, D. R., et al. 2012, J. Phys. B At. Mol. Phys., 45, 235201 


\section{Appendix A: Fitting the $\boldsymbol{n}$ distributions}

It has long been known that the CX cross section often distributes continuously as a function of $n$ with a maximum near $n_{\mathrm{p}}$, when the ion charge $q$ is sufficiently large (see Janev \& Winter 1985 for a review). To quantify the $n$ distribution, we compiled the velocity- and $n$ - dependent cross sections found in literature in Sect. 3.2 and fit them with a phenomenological third-degree polynomial curve,

$$
\begin{aligned}
\lg \sigma_{\text {norm }}(n)= & c_{1}(v)+c_{2}(v) n_{\text {norm }}(n, q)+c_{3}(v) n_{\text {norm }}^{2}(n, q) \\
& +c_{4}(v) n_{\text {norm }}^{3}(n, q),
\end{aligned}
$$

where $\sigma_{\text {norm }}(n)$ is the capture fraction into $n, c_{1}(v)$ to $c_{4}(v)$ are four velocity-dependent fitting parameters, and $n_{\text {norm }}(n, q)=(n-$ $\left.n_{\mathrm{p}}\right) / n_{\mathrm{p}}$. The best-fit parameters are shown in Table A.1.
Table A.1. $n$-distribution fitting parameters

\begin{tabular}{lcccc}
\hline \hline$v\left(\mathrm{~km} \mathrm{~s}^{-1}\right)$ & $c_{1}(v)^{a}$ & $c_{2}(v)^{a}$ & $c_{3}(v)^{a}$ & $c_{4}(v)^{a}$ \\
\hline 50 & -0.46 & 10.79 & -60.37 & -37.08 \\
80 & -0.37 & 9.38 & -58.85 & -18.09 \\
120 & -0.40 & 9.29 & -48.03 & -33.64 \\
200 & -0.31 & 7.73 & -44.56 & -21.31 \\
350 & -0.21 & 5.68 & -37.53 & -14.48 \\
600 & -0.17 & 3.39 & -30.87 & -0.82 \\
900 & -0.24 & 1.49 & -14.59 & -18.47 \\
1300 & -0.30 & 1.16 & -10.12 & -10.05 \\
1800 & -0.30 & 0.58 & -7.95 & -0.32 \\
2400 & -0.36 & 0.64 & -6.75 & 4.11 \\
3100 & -0.34 & 0.70 & -4.60 & -2.77 \\
3900 & -0.37 & 0.84 & -4.06 & -7.24 \\
4800 & -0.39 & 0.83 & -4.06 & -10.45 \\
5800 & -0.40 & 0.63 & -3.90 & -10.66 \\
\hline
\end{tabular}

Notes. ${ }^{(a)}$ Parameters of the polynomial function (Eq. (A.1)). 
L. Gu et al.: New charge exchange model

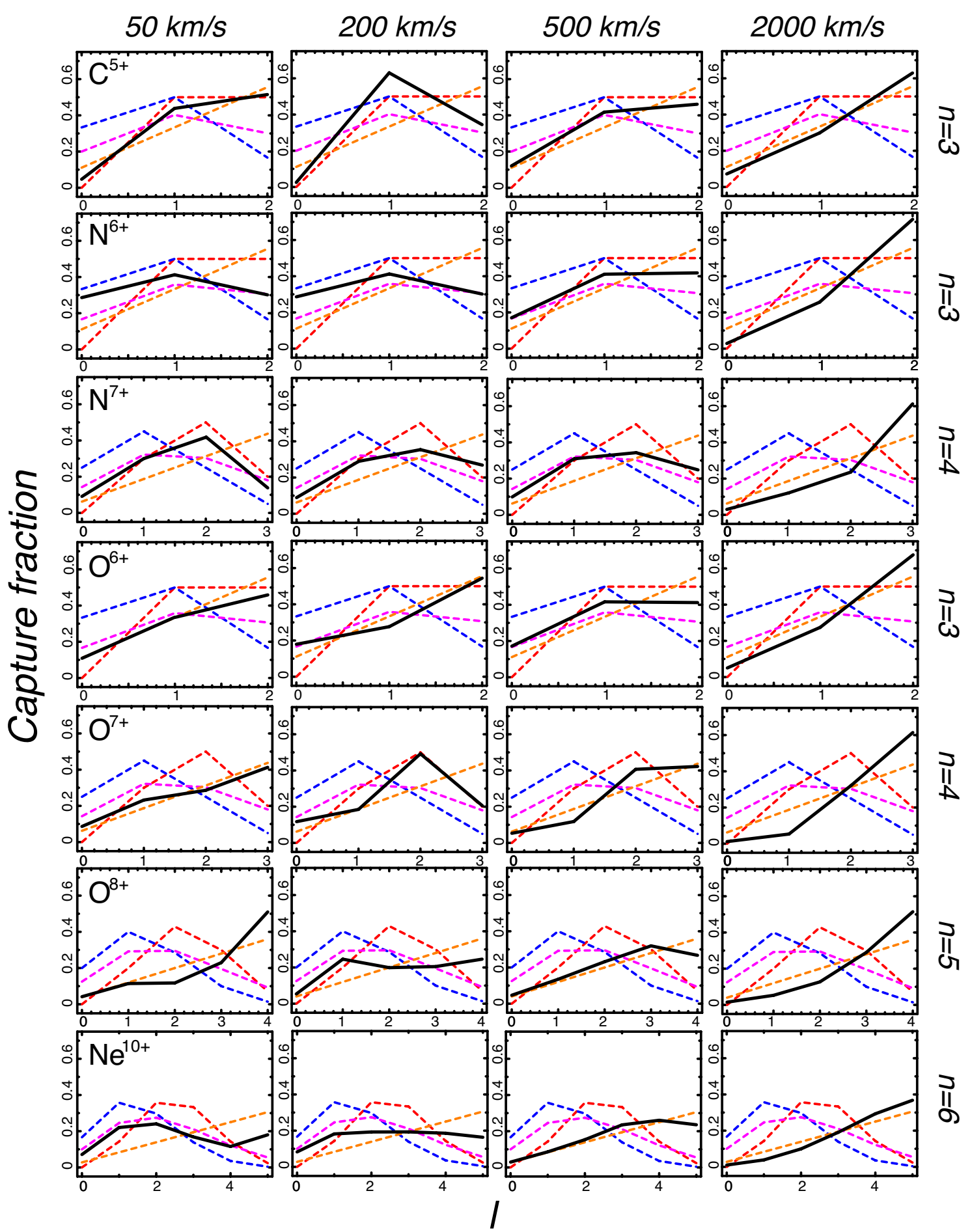

Fig. A.1. Averaged fractions of $l$-dependent capture for $\mathrm{C}^{5+}, \mathrm{N}^{6+}, \mathrm{N}^{7+}, \mathrm{O}^{6+}, \mathrm{O}^{7+}, \mathrm{O}^{8+}$, and $\mathrm{Ne}^{10+}$, plotted as a function of $l$. For each ion, the dominant $n$-shell in $n \leq n_{\mathrm{p}}$ is shown. The color and line styles are the same as in Fig. 3 . 


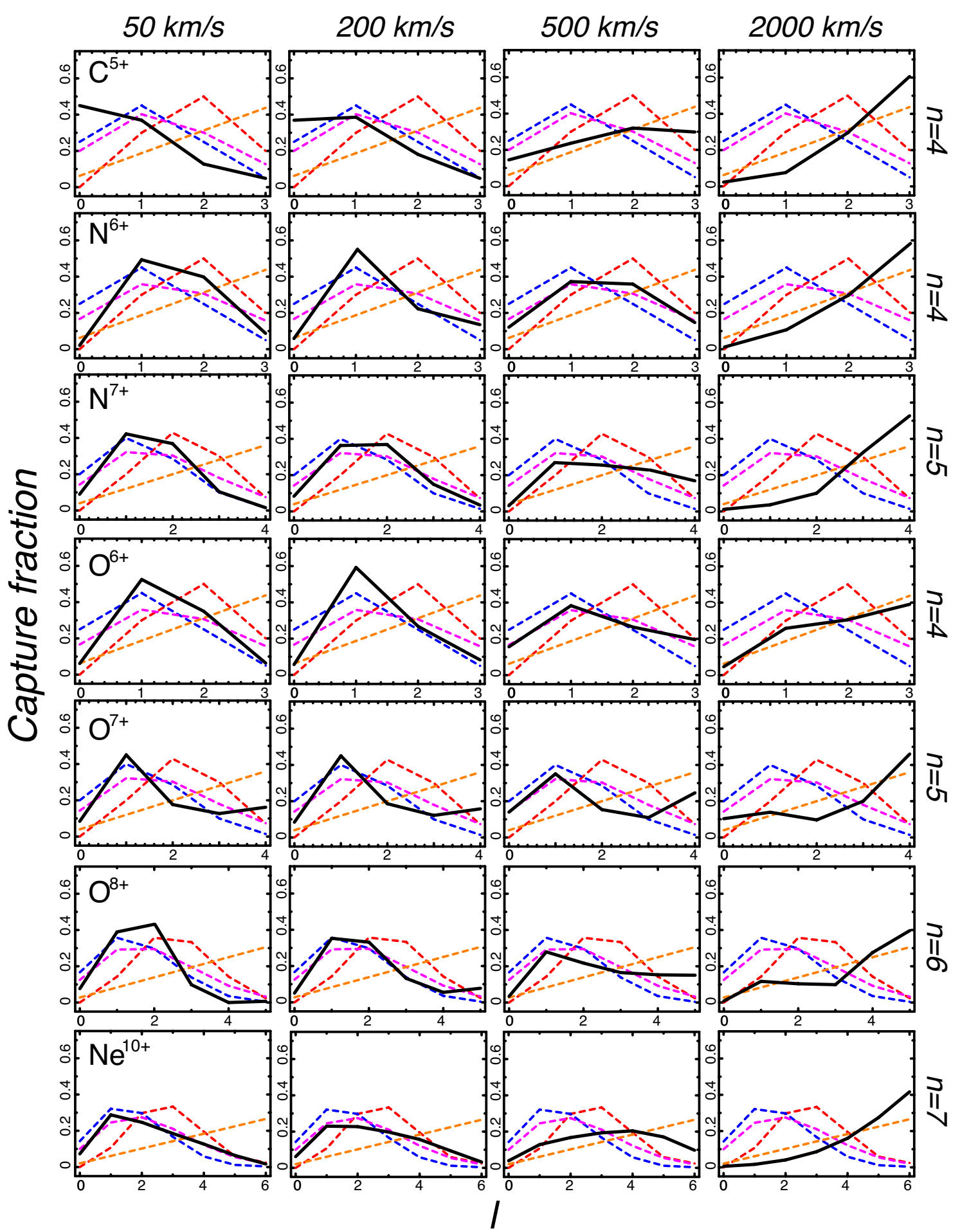

Fig. A.2. Averaged fractions of $l$-dependent capture for $\mathrm{C}^{5+}, \mathrm{N}^{6+}, \mathrm{N}^{7+}, \mathrm{O}^{6+}, \mathrm{O}^{7+}, \mathrm{O}^{8+}$, and $\mathrm{Ne}^{10+}$, plotted as a function of $l$. For each ion, the dominant $n$-shell in $n>n_{\mathrm{p}}$ is shown. The color and line styles are the same as in Fig. 3. 


\section{Appendix B: Preferred I-distributions}

Here we describe in detail a velocity-dependent scheme to approximate the $l$-selectivity. As shown in Table 1 , the $l$-resolved cross section data, derived from theoretical calculation, are available for reactions with the seven ions, i.e., $\mathrm{C}^{5+}, \mathrm{N}^{6+}, \mathrm{N}^{7+}, \mathrm{O}^{6+}$, $\mathrm{O}^{7+}, \mathrm{O}^{8+}$, and $\mathrm{Ne}^{10+}$. For other ions, the five canonical weighting functions, as shown in Eqs. (4)-(8), were utilized as follows.

For each velocity, we determined the preferred weighting function by comparing them to the available data. As described in Sect. 3.3, the preferred function must be chosen separately for shells with principle quantum number $n \leq n_{\mathrm{p}}$ and those with $n>n_{\mathrm{p}}$. Here we only study the most dominant shell in each group (Figs. A.1 and A.2). For $n \leq n_{\mathrm{p}}$, the $W^{\text {se }}$ function (Eq. (6)) is recommended at low velocities, i.e., $v=50$ and $200 \mathrm{~km} \mathrm{~s}^{-1}$, while the statistical weight $W^{\text {st }}$ becomes more popular in the intermediate- and high- velocity regimes $(v=500$ and $2000 \mathrm{~km} \mathrm{~s}^{-1}$ ). As shown in Fig. A.1, this scheme can be applied approximately to most reactions, except for a few outliers, such as $\mathrm{C}^{5+}$ and $\mathrm{O}^{8+}$ at $v=50 \mathrm{~km} \mathrm{~s}^{-1}, \mathrm{O}^{6+}$ at $v=200 \mathrm{~km} \mathrm{~s}^{-1}$, and $\mathrm{N}^{7+}$ at $v=500 \mathrm{~km} \mathrm{~s}^{-1}$. As for $n>n_{\mathrm{p}}$, the $l$-distribution is represented best by $W^{\mathrm{L} 2}$ at $v=50$ and $200 \mathrm{~km} \mathrm{~s}^{-1}, W^{\text {se }}$ at $v=500 \mathrm{~km} \mathrm{~s}^{-1}$, and $W^{\text {st }}$ at $v=2000 \mathrm{~km} \mathrm{~s}^{-1}$, albeit with a few exceptions such as $\mathrm{C}^{5+}$ and $\mathrm{Ne}^{10+}$ at $v=500 \mathrm{~km} \mathrm{~s}^{-1}$.

To define the $l$-preference continuous in the velocity space, we further analyzed the data with a finer velocity grid of 20 points. It is found that the $l$-distributions for $n \leq n_{\mathrm{p}}$ shells mostly switch at $v=500 \mathrm{~km} \mathrm{~s}^{-1}$ from a $W^{\text {se }}$ form to a $W^{\text {st }}$ form, while the $n>n_{\mathrm{p}}$ shells are likely to evolve from $W^{12}$ to $W^{\text {se }}$ at $v=300 \mathrm{~km} \mathrm{~s}^{-1}$, and from $W^{\text {se }}$ to $W^{\text {st }}$ at $v=500 \mathrm{~km} \mathrm{~s}^{-1}$.

\section{Appendix C: More features of the SPEX-CX model}

Our plasma code for CX emission is included as an independent model in the SPEX package (Kaastra et al. 1996) ${ }^{2}$. The model first calculates the fraction of each ion at a ionization temperature $T_{\mathrm{i}}$ and element abundance $A$, then evaluates the CX spectrum for a collision velocity $v$ and emission measure norm. The rate coefficients obtained in Sect. 3 are fully utilized in the model, and the actual data are tabulated in the form of FITS files ${ }^{3}$ in the SPEX database. They will be updated with more recent published results once these become available.

The CX model contains three additional parameters for different physical conditions. First, the collision velocity $v$ can be replaced by the velocity of random thermal motion, which is characterized by ion temperature $T_{i}$. This is appropriate for some hot plasmas where thermal motion dominates. Second, besides the single collision mode, our model also allows multiple collisions between ions and neutrals. In the latter case, one ion would continuously undergo $\mathrm{CX}$ and produce various emission lines, until it becomes neutral. This is suited more to objects with dense neutral materials. Finally, the model provides five types of l-weighting functions (Eqs. (4)-(8)) for the CX cross section. The optimized method is described in Sect. 3.3, while the five basic functions can also be selected to fine-tune the spectrum and to test the sensitivity of data to the assumed subshell populations.

\footnotetext{
2 https://www.sron.nl/spex

3 http://fits.gsfc.nasa.gov/fits_home.html
} 